\title{
$K-\epsilon-L$ model in turbulent superfluid helium
}

\author{
Michele Sciacca $^{1} \quad$ David Jou ${ }^{2,3} \quad$ Maria Stella Mongiovì \\ ${ }^{1}$ Dipartimento di Scienze Agrarie e Forestali, Università di Palermo, Viale delle Scienze, 90128 Palermo, Italy \\ ${ }^{2}$ Departament de Física, Universitat Autònoma de Barcelona, 08193 Bellaterra, Catalonia, Spain \\ ${ }^{3}$ Institut d'Estudis Catalans, Carme 47, Barcelona 08001, Catalonia, Spain
}

\begin{abstract}
We generalize the $K-\epsilon$ model of classical turbulence to superfluid helium. In a classical viscous fluid the phenomenological eddy viscosity characterizing the effects of turbulence depends on the turbulent kinetic energy $K$ and the dissipation function $\epsilon$, which are mainly related to the fluctuations of the velocity field and of its gradient. In superfluid helium, instead, we consider the necessary coefficients for describing the effects of classical and quantum turbulence, involving fluctuations of the velocity, the heat flux, and the vortex line density of the quantized vortex lines. By splitting the several fields into a timeaverage part and a fluctuating part, some expressions involving the second moments of the turbulent fluctuations appear in the evolution equations for the average quantities. As in the $K-\epsilon$ model, a practical way of closing such equations is to tentatively express such fluctuating terms as a function of the average quantities. In this context we propose how the turbulent coefficients so introduced could depend on the second moments of the fluctuations of $\mathbf{v}, \mathbf{q}$ and $L$ (respectively denoted as $K, K_{q}$ and $K_{L}$ ), and on their respective dissipation functions (related to the second moments of their gradients) $\epsilon, \epsilon_{q}$ and $\epsilon_{L}$.
\end{abstract}

Keywords: $K-\epsilon$ model; superfluid helium; heat transfer; quantum turbulence; energy cascade; quantized vortices; non-equilibrium thermodynamics.

\section{Introduction}

The study of turbulence is an appealing topic which has interested many researchers in different fields, from the smallest scalles to the largest ones, from nanosystems to universe. The methods and approaches used for dealing with all the phenomena involved into turbulence span from hydrodynamics to thermodynamics and statistics $[1,2,3,4,5,6,7,8,9,10,11,12,13]$.

Here we are interested in the $K-\epsilon$ model as the zero-th order closure model in the hierarchy of the moments of the fluctuations of the main fields, usually applied in a viscous classical fluid [14]. In this paper we aim at generalizing this method to superfluid helium, where an additional different kind of turbulence occurs: quantum turbulence. Quantum turbulence is typically thought as a tangle of quantized vortex lines, which are characterized by a quantized circulation $\kappa=h / m$ ( $h$ and $m$ being the Planck's constant and the helium atom mass, respectively), a fixed core of radius of the order of the size of the helium atom, and a vortex length density per unit volume $L$.

\footnotetext{
${ }^{0}$ E-mail addresses: michele.sciacca@unipa.it (M. Sciacca), david.jou@uab.cat (D. Jou), m.stella.mongiovi@unipa.it (M. S. Mongiovì).
} 
There are still many open questions about the existence and interaction of both kinds of turbulence (classical and quantum) in superfluid helium. Indeed, superfluid helium is usually thought as composed of two indintinguishable components, the normal component (a viscous fluid which carries the entropy and the viscosity of superfluid helium) and an inviscid superfluid component. According to the two-fluid model, proposed by Tisza and Landau [15, 16], quantized vortices are caused by a vorticity of the superfluid component. Recently, in [17] it was argued that the normal component could be also turbulent, and it would explain the presence of two regimes of turbulence in counterflow experiments. An alternative explanation could be that an inhomogeneous and locally polarized vortex tangle (regime TI) becomes an homogeneous state (regime TII) for enhanced applied heat flux, because of the breakdown of these localized polarizations [18]. The understanding of the phenomena is still open, but it points to different kinds of turbulence for the normal component with speed $\mathbf{v}_{n}$, the superfluid component with speed $\mathbf{v}_{s}$, and the vortex tangle itself. The last feature has been studied from the numerical point of view, and it is seen that at very low temperature (where the normal component is practically absent), quantized vortices may be gathered into bundles of vortices, which mimic classical eddies [19, 20, 21, 22].

From the thermodynamical point of view, an alternative model of superfluid helium was proposed by means of the Extended Thermodynamics [23, 24], which takes the heat flux q as further independent field than the usual mean velocity $\mathbf{v}$. In this paper we consider $\mathbf{v}$ and $\mathbf{q}$ (rather than $\mathbf{v}_{n}$ and $\mathbf{v}_{s}$ ) together with $L$ as the main independent quantities.

By splitting the several fields into a time-average part and a fluctuating part, some expressions involving the second moments of the turbulent fluctuations in $\mathbf{v}^{\prime}, \mathbf{q}^{\prime}$ and $L^{\prime}$ appear in the evolution equations for the average quantities $\overline{\mathbf{v}}, \overline{\mathbf{q}}$ and $\bar{L}$. In the usual $K-\epsilon$ model for classical fluids, the way of closing the evolution equation for $\overline{\mathbf{v}}$ is to tentatively express the second moments of the fluctuating terms of $\mathbf{v}^{\prime}$ as a function of gradient of $\mathbf{v}$, in such a way that the final equations have a form analogous to the usual equations for $\mathbf{v}$, but with effective kinematic viscosity which is a sum of the molecular contribution and the turbulent contribution (the so-called eddy viscosity). In its turn, this turbulent contribution is expressed in terms of some quantities related to turbulent fluctuations, as the kinetic energy of fluctuations, $K$, and the dissipation function $\epsilon$. In the context of the present paper, we analize the equations, identify the turbulent contributions, propose for them some turbulent transport coefficients, and try to express them in terms of the second moments $\mathbf{v}$, $\mathbf{q}$ and $L$, namely $K, K_{q}$ and $K_{L}$, as well as of their corresponding dissipation functions $\epsilon, \epsilon_{q}$ and $\epsilon_{L}$, related to the second-order moments of their gradients. Since some of the details may depend on the kind of flow, we do not pretend that this model will grasp all the complexity of turbulence, but it is logical to take it into consideration as a macroscopic starting point, in analogy to the $K-\epsilon$ model in classical turbulence $[1,4,8,9,10,11,14]$.

The paper is organized as follows: in Section 2 we deal with the basic equations of the onefluid extended model of superfluid helium and their time-averaged expressions; in Section 3 we consider the zero-th order approximation closure and we propose some expressions to close the equations of the $K-\epsilon-L$ model for $\mathbf{v}$ and $\mathbf{q}$; in Section 4 we deal with the equation for $L$; Section 5 is devoted to the results and the concluding remarks of the paper. 


\section{The one-fluid extended model: basic equations and their versions for turbulent flows}

In this section we consider the basic equations of the one-fluid extended model for superfluid helium. The interest to deal with this model instead of the two-fluid model is based mainly on the use of the fields directly measurable in the experiments (heat flux $\mathbf{q}$ and mean velocity v) and on the idea that the extended model can be used also in the ballistic regime (which occurs at very low temperature or in very thin channels), as shown in Ref. [25].

\subsection{Basic equations of the one-fluid extended model}

The main fields of the one-fluid extended model are the mass density $\rho$, the velocity $\mathbf{v}$, the heat flux q, the energy density $u$, and the vortex line density per unit volume $L$. The field equations of $\mathrm{He}$ II in the presence of dissipation, neglecting body forces, are the following $[26,27,18]$ :

$$
\left\{\begin{array}{l}
\dot{\rho}+\rho \nabla \cdot \mathbf{v}=0 \\
\rho \dot{\mathbf{v}}+\nabla p+\nabla \cdot \mathbf{P}_{\mathbf{v}}=0 \\
\rho \dot{u}+\nabla \cdot \mathbf{q}+p \nabla \cdot \mathbf{v}-\mathbf{P}_{\mathbf{v}}: \nabla \mathbf{v}=0, \\
\dot{\mathbf{q}}+\zeta \nabla T+\zeta \nabla \cdot \mathbf{P}_{\mathbf{q}}=\sigma^{\mathbf{q}}=-K_{f} L \mathbf{q}, \\
\dot{L}-D_{L} \nabla^{2} L=-\beta \kappa L^{2}+\alpha q L^{3 / 2},
\end{array}\right.
$$

with $p$ the pressure, $T$ the temperature, $\zeta=\lambda_{1} / \tau_{1}$ ( $\lambda_{1}$ being the heat conductivity of He II and $\tau_{1}$ the relaxation time of the heat flux), that characterizes the second-sound velocity [18], $K_{f}=\frac{1}{3} B_{H V} \kappa$ (where $B_{H V}$ is the Hall-Vinen coefficient of the mutual friction strength), $\alpha$ and $\beta$ are numerical constants related to the production and destruction of quantum vortices, respectively, according to the well-known Vinen equation, $q$ in the last equation is the modulus of the heat flux, and $\mathbf{P}_{\mathbf{v}}$ and $\mathbf{P}_{\mathbf{q}}$ are non-equilibrium stress tensor of momentum and heat flux, given by:

$$
\begin{aligned}
& \mathbf{P}_{\mathbf{v}}=-2 \eta\left[\langle\nabla \mathbf{v}\rangle+\frac{1}{\rho s T}\langle\nabla \mathbf{q}\rangle\right] \\
& \mathbf{P}_{\mathbf{q}}=-\frac{2}{\rho s T} \eta\left[\langle\nabla \mathbf{v}\rangle+\frac{1}{\rho s T}\langle\nabla \mathbf{q}\rangle\right] .
\end{aligned}
$$

where $\eta$ is the shear viscosity and $s$ is the entropy density. The bulk viscosity has been

neglected. In the equations (2.1) a "dot" over the main fields stands for $\frac{\partial}{\partial t}+\mathbf{v} \cdot \nabla$ and $\nabla$ standing for the gradient operator. The term $-D_{L} \nabla^{2}$ in the last equation of the system (2.1) takes vortex diffusion into account [18, 28, 29, 30, 31].

Let's assume that the mass density is constant, namely $\dot{\rho}=0$, which yields $\nabla \cdot \mathbf{v}=0$ and assume an isothermal situation, in which the energy balance equation is not necessary. Then, substituting $\mathbf{P}_{\mathbf{v}}$ and $\mathbf{P}_{\mathbf{q}}$ in the equation (2.4b) and (2.4c) and assuming that $\rho s T$ in the expression of $\mathbf{P}_{\mathbf{v}}$ and of $\mathbf{P}_{\mathbf{q}}$ does not depend on the spatial coordinates, we are left with: 


$$
\left\{\begin{array}{l}
\frac{\partial}{\partial t} \mathbf{v}+\mathbf{v} \cdot \nabla \mathbf{v}+\frac{1}{\rho} \nabla p-\nu \nabla^{2} \mathbf{v}-\frac{\nu}{\rho s T} \nabla^{2} \mathbf{q}=0 \\
\frac{\partial}{\partial t} \mathbf{q}+\mathbf{v} \cdot \nabla \mathbf{q}+\zeta \nabla T-\mu \nabla^{2} \mathbf{v}-\frac{\mu}{\rho s T} \nabla^{2} \mathbf{q}=-K_{f} L \mathbf{q} \\
\frac{\partial}{\partial t} L+\mathbf{v} \cdot \nabla L-D_{L} \nabla^{2} L=-\beta \kappa L^{2}+\alpha q L^{3 / 2}
\end{array}\right.
$$

where $\nu=\eta / \rho$ is the classical kinematic viscosity (length ${ }^{2} /$ time) and $\mu=\frac{\nu \zeta}{s}$ is the "heat" kinematic viscosity (length ${ }^{4} \mathrm{Kg} /$ time $^{3}$ ). The two first equations in (2.4) suggest to define two kinds of thermal viscosity, namely $\nu_{1} \equiv \frac{\nu}{\rho s T}($ time $/ \mathrm{kg})$ and $\mu_{1} \equiv \frac{\mu}{\rho s T}($ length $2 /$ time , which will be useful for the further discussions.

The last equation in (2.4) is the equation for the vortex line density $L$, which was proposed for the first time by Vinen without the inertial term and then generalized by many authors. It has been extended to cover the diversity of the phenomenologies, to include the influence of the walls $[18,32]$, the anisotropy of the vortex tangle [33], the influence of the transverse flow [34] and other situations of interest as in Ref. [35, 36, 37]. However, in this paper we have preferred do not take the most general equation but the simplest form for the source term (the Vinen equation) with the inertial term on the left side.

\subsection{Time-averaged evolution equations of the main fields $\mathrm{v}, \mathrm{q}$ and $L$}

To discuss turbulence we write $\mathbf{v}=\overline{\mathbf{v}}+\mathbf{v}^{\prime}, \mathbf{q}=\overline{\mathbf{q}}+\mathbf{q}^{\prime}, p=\bar{p}+p^{\prime}, T=\bar{T}+T^{\prime}$, and $L=\bar{L}+L^{\prime}$, with $\overline{\mathbf{v}}, \bar{u}, \bar{p}, \bar{T}$ and $\bar{L}$ the time average values and $\mathbf{v}^{\prime}, p^{\prime}, T^{\prime}$ and $L^{\prime}$ the corresponding fluctuating turbulent contributions. For the sake of simplicity we will assume that the density does not fluctuate and that it has the constant value $\rho$.

Because of the linearity of the continuity equation $\nabla \cdot \mathbf{v}=0$, we find $\nabla \cdot \overline{\mathbf{v}}=0$ and

$$
\nabla \cdot \mathbf{v}^{\prime}=0
$$

Let's consider now the equation of the velocity $\mathbf{v}$, namely equation (2.4b). By the above substitutions, it becomes

$$
\frac{\partial}{\partial t} \overline{\mathbf{v}}+(\overline{\mathbf{v}} \cdot \nabla) \overline{\mathbf{v}}+\overline{\left(\mathbf{v}^{\prime} \cdot \nabla\right) \mathbf{v}^{\prime}}+\frac{1}{\rho} \nabla \bar{p}-\nu \nabla^{2} \overline{\mathbf{v}}-\nu_{1} \nabla^{2} \overline{\mathbf{q}}=0
$$

and by means of the identity $\left(\mathbf{v}^{\prime} \cdot \nabla\right) \mathbf{v}^{\prime}=\nabla \cdot\left(\mathbf{v}^{\prime} \otimes \mathbf{v}^{\prime}\right)-\left(\nabla \cdot \mathbf{v}^{\prime}\right) \mathbf{v}^{\prime}$ and (2.5) we obtain

$$
\frac{\partial}{\partial t} \overline{\mathbf{v}}+(\overline{\mathbf{v}} \cdot \nabla) \overline{\mathbf{v}}+\nabla \cdot \overline{\mathbf{v}^{\prime} \otimes \mathbf{v}^{\prime}}+\frac{1}{\rho} \nabla \bar{p}-\nu \nabla^{2} \overline{\mathbf{v}}-\nu_{1} \nabla^{2} \overline{\mathbf{q}}=0
$$

We follow the same procedure for equation (2.4c) for $\mathbf{q}$ and obtain:

$$
\frac{\partial}{\partial t} \overline{\mathbf{q}}+(\overline{\mathbf{v}} \cdot \nabla) \overline{\mathbf{q}}+\nabla \cdot \overline{\mathbf{v}^{\prime} \otimes \mathbf{q}^{\prime}}+\zeta \nabla \bar{T}-\mu \nabla^{2} \overline{\mathbf{v}}-\mu_{1} \nabla^{2} \overline{\mathbf{q}}=-K_{f} \bar{L} \overline{\mathbf{q}}-K_{f} \overline{L^{\prime} \mathbf{q}^{\prime}}
$$

where we have used the identity $\left(\mathbf{v}^{\prime} \cdot \nabla\right) \mathbf{q}^{\prime}=\nabla \cdot\left(\mathbf{v}^{\prime} \otimes \mathbf{q}^{\prime}\right)-\left(\nabla \cdot \mathbf{v}^{\prime}\right) \mathbf{q}^{\prime}=\nabla \cdot\left(\mathbf{v}^{\prime} \otimes \mathbf{q}^{\prime}\right)$ because of $(2.5)$. 
Note that in equations (2.7) and (2.8) there are three terms dependent on the second moments of $\mathbf{v}^{\prime}, \mathbf{q}^{\prime}$ and $L^{\prime}$, namely $\mathbf{v}^{\prime} \otimes \mathbf{v}^{\prime}, \mathbf{v}^{\prime} \otimes \mathbf{q}^{\prime}$ and $L^{\prime} \mathbf{q}^{\prime}$. To close these equations, expressions for these contributions in terms of $\overline{\mathbf{v}}, \overline{\mathbf{q}}$ and $\bar{L}$ must be provided.

Regarding the equation (2.4) for the vortex line density $L$, the right-hand side is the source term, which is made by two terms, empirically proposed by Vinen and confirmed analytically by Schwarz [38]. The first term $\left(\beta \kappa L^{2}\right)$ refers to the decay of vortex line density whereas the second term $\left(\alpha q L^{3 / 2}\right)$ refers to the production of vortex line density. Since $\ell=L^{-1 / 2}$ is the inter-vortex space (namely, the average separation between neighboring vortices) and it is usually the scale where the interaction between vortices becomes stronger, we rewrite the right-hand side of the equation of $L$ in terms of $\mathcal{L}=L^{1 / 2}=\ell^{-1}$, namely the reciprocal of the scale at which transfer of energy is of remarkable importance. Thus, the equation becomes:

$$
\frac{\partial}{\partial t} L+\mathbf{v} \cdot \nabla L-D_{L} \nabla^{2} L=-\beta \kappa \mathcal{L}^{4}+\alpha q \mathcal{L}^{3}
$$

where the production term can be also seen as $\alpha q \frac{L}{\ell}$ and the destruction term as $\beta \kappa \frac{L}{\ell^{2}}$. This will be useful for our discussion in Section 4.

Inserting the fluctuations in the equation (2.9) for the evolution equation for $\bar{L}$ we find:

$$
\begin{gathered}
\frac{\partial}{\partial t} \bar{L}+\overline{\mathbf{v}} \cdot \nabla \bar{L}+\overline{\mathbf{v}^{\prime} \cdot \nabla L^{\prime}}-D_{L} \nabla^{2} \bar{L}=\alpha \bar{q}\left(\overline{\mathcal{L}^{3}}+3 \overline{\mathcal{L}} \overline{\mathcal{L}^{\prime 2}}+\overline{\mathcal{L}^{\prime 3}}\right)+ \\
-\beta \kappa\left(\overline{\mathcal{L}^{4}}+6 \overline{\mathcal{L}^{2}} \overline{\mathcal{L}^{\prime 2}}+4 \overline{\mathcal{L}} \overline{\mathcal{L}^{\prime 3}}+\overline{\mathcal{L}^{\prime 4}}\right)+\alpha\left(3 \overline{\mathcal{L}^{2}} \overline{q^{\prime} \mathcal{L}^{\prime}}+3 \overline{\mathcal{L}} \overline{q^{\prime} \mathcal{L}^{\prime 2}}+\overline{q^{\prime} \mathcal{L}^{\prime 3}}\right)
\end{gathered}
$$

where we have considered $L=\bar{L}+L^{\prime}, \mathcal{L}=\overline{\mathcal{L}}+\mathcal{L}^{\prime}$. The above equation can be also written:

$$
\begin{gathered}
\frac{\partial}{\partial t} \bar{L}+\overline{\mathbf{v}} \cdot \nabla \bar{L}+\nabla \cdot \overline{\mathbf{v}^{\prime} L^{\prime}}-D_{L} \nabla^{2} \bar{L}=\alpha q\left(\overline{\mathcal{L}^{3}}+3 \overline{\mathcal{L}} \overline{\mathcal{L}^{\prime 2}}+\overline{\mathcal{L}^{\prime 3}}\right)+ \\
-\beta \kappa\left(\overline{\mathcal{L}^{4}}+6 \overline{\mathcal{L}^{2}} \overline{\mathcal{L}^{\prime 2}}+4 \overline{\mathcal{L}} \overline{\mathcal{L}^{\prime 3}}+\overline{\mathcal{L}^{\prime 4}}\right)+\alpha\left(3 \overline{\mathcal{L}^{2}} \overline{q^{\prime} \mathcal{L}^{\prime}}+3 \overline{\mathcal{L}} \overline{q^{\prime} \mathcal{L}^{\prime 2}}+\overline{q^{\prime} \mathcal{L}^{\prime 3}}\right)
\end{gathered}
$$

because of the identity $\mathbf{v}^{\prime} \cdot \nabla L^{\prime}=\nabla \cdot\left(\mathbf{v}^{\prime} L^{\prime}\right)-\left(\nabla \cdot \mathbf{v}^{\prime}\right) L^{\prime}=\nabla \cdot\left(\mathbf{v}^{\prime} L^{\prime}\right)$. In $(2.11)$, terms in the fluctuations of $\mathcal{L}^{\prime}$ and $\mathbf{q}^{\prime}$ appear. As for (2.7) and (2.8), expressions for these contributions in terms of $\overline{\mathbf{v}}$ and $\overline{\mathbf{q}}$ and $\bar{L}$ must be provided.

In fact, from a physical point of view, the most complex and controversial part of the modelization refers to the fluctuations of vortex line density, which goes deeper into open problems. In Ref. [39], Nemirovskii deals with the fluctuation spectrum of $L$ in the counterflowing turbulence (Vinen equation), where vortices are mostly loops uniformly distributed in the fluid; and in the quasi-classical turbulence, where vortices forms vortex bundles. It turns out that the fluctuation spectrum depends on the kind of flow. Thus, a completely universal modelization would not be possible in simple terms. We will comment later on this limitation in the concluding remarks.

\section{Closure relations for $\overline{\mathrm{v}}$ and $\overline{\mathrm{q}}$ : zero-th order approximation}

Now, we define with specific symbols the terms involving moments of the fluctuations in the evolution equations for $\overline{\mathbf{v}}(2.7)$ and $\overline{\mathbf{q}}(2.8)$, namely $\mathbf{R}_{v} \equiv \overline{\mathbf{v}^{\prime} \otimes \mathbf{v}^{\prime}}$ appearing in (2.7) and $\mathbf{R}_{q} \equiv \overline{\mathbf{v}^{\prime} \otimes \mathbf{q}^{\prime}}$ and $\mathbf{R}_{q L} \equiv K_{f} \overline{\mathbf{q}^{\prime} L^{\prime}}$ appearing in (2.8) (in Section 4 we will consider the evolution equation for $\bar{L})$. In order to close the equations, these quantities must be given in terms of the 
average quantities. Alternatively, an evolution equation for them should be found by averaging the equations for $\mathbf{v}^{\prime} \otimes \mathbf{v}^{\prime}, \mathbf{v}^{\prime} \otimes \mathbf{q}^{\prime}$ and $\mathbf{q}^{\prime} L^{\prime}$. In such equations, third-order moments of the form $\mathbf{v}^{\prime} \otimes \mathbf{v}^{\prime} \otimes \mathbf{v}^{\prime}, \mathbf{v}^{\prime} \otimes \mathbf{v}^{\prime} \otimes \mathbf{q}^{\prime}, \mathbf{v}^{\prime} \otimes \mathbf{q}^{\prime} \otimes L^{\prime}$ and so on, as well as second-order moments of the spatial gradients of fluctuations would appear, thus, leading to a hierarchy of higher-order moments, in which moments of order $n+1$ appear in the evolution equation of moments of order $n$.

\subsection{A short remainder of the $K-\epsilon$ model}

In classical turbulence it is assumed that the extra turbulent contribution to the evolution equation for $\overline{\mathbf{v}}$, namely the third term of equation (2.7), has a form analogous to that of the usual viscous term, but with an effective viscosity of turbulent origin. Thus, it is taken $[9,10,11,14]$

$$
\mathbf{R}_{v} \equiv \overline{\mathbf{v}^{\prime} \otimes \mathbf{v}^{\prime}}=-2 \nu_{t} \nabla^{s} \overline{\mathbf{v}}
$$

where the Boussinesq's eddy viscosity assumption is assumed with $\nu_{t}$ the so-called eddyviscosity, which phenomenologically describes the contribution of the turbulence to the hydrodynamic resistance to the flow, and $\nabla^{s} \overline{\mathbf{v}}$ the strain rate tensor defined as $\nabla^{s} \overline{\mathbf{v}}=\left(\nabla \overline{\mathbf{v}}+\nabla \overline{\mathbf{v}}^{T}\right) / 2$. The motivation of such closure is keeping the form of the Navier-Stokes equation, but with a phenomenologically modified viscosity, describing the big increase of the dissipation in turbulent flow as composed to the corresponding laminar flows. Such eddy vorticity is assumed to depend on turbulent features, namely the kinetic energy $K$ per unit mass, $K=\frac{1}{2} \overline{\mathbf{v}^{\prime} \cdot \mathbf{v}^{\prime}}$ and $\epsilon$ the dissipation function $\epsilon=2 \nu \overline{\nabla^{s} \mathbf{v}^{\prime}: \nabla^{s} \mathbf{v}^{\prime}}$, which naturally appears as in equation (3.12) (see the reference $[\mathbf{9}, \mathbf{1 0}, \mathbf{1 1}, \mathbf{1 4}])$. The dimensions of $K$ are $\left(\right.$ length $\left.^{2} \times \operatorname{time}^{-2}\right)$ and those of $\epsilon$ are $\left(\right.$ length $^{2} \times$ time $^{-3}$ ), thus, the combination of $K$ and $\epsilon$ with the suitable dimensions of the eddy viscosity $\left(\right.$ length $^{2} \times$ time $\left.^{-1}\right)$ is

$$
\nu_{t}=C_{\nu} \frac{K^{2}}{\epsilon}
$$

with $C_{\nu}$ a dimensionless constant which is of the order of 0.09 [40]. With (3.1) and (3.2), the evolution equation (2.7) for $\overline{\mathbf{v}}$ in a classical fluid (i.e. with $\overline{\mathbf{q}}=0$ ), becomes closed apart from the field $\epsilon$. Evolution equations for $K$ and $\epsilon$ are proposed below in (3.14) and (3.18). The closure of the hierarchy of higher-order moments is crucial for modeling and understanding the main features of turbulence, and there are many different strategies of truncating this hierarchy: use of maximum-entropy methods [11], [41], [4], [8], [42], [43], [44], [45], [46], [47]; or analogies with well-known models and methods of the Kinetic theory of gases [48, 49, 14, 50] based on some generalized forms of Boltzmann equation or of lattice-Boltzmann equation [51], $[52]$.

\subsection{A generalized $K-\epsilon-L$ model in superfluid helium}

\subsubsection{Closure of equations for $\bar{v}$ and $\bar{q}$}

In this section we will take a lowest-order closure of equations (2.7) and (2.8) by expressing $\mathbf{R}_{v}, \mathbf{R}_{q}, \mathbf{R}_{q L}$ in terms of $\overline{\mathbf{v}}, \overline{\mathbf{q}}$ and $\overline{\mathbf{L}}$ in such a way that they have a form similar to (2.4a) and $(2.4 \mathrm{~b})$ but with new coefficients related to turbulence. As in (3.1) this allows that the equations for $\overline{\mathbf{v}}$ and $\overline{\mathbf{q}}$ have the same form as those for $\mathbf{v}$ and $\mathbf{q}$, but with effective turbulent parameters. Generalizing (3.1) we propose the following expressions: 


$$
\begin{gathered}
\mathbf{R}_{v} \equiv \overline{\mathbf{v}^{\prime} \otimes \mathbf{v}^{\prime}}=-\nu_{t} \nabla \overline{\mathbf{v}}-\nu_{1 t} \nabla \overline{\mathbf{q}} \\
\mathbf{R}_{q} \equiv \overline{\mathbf{v}^{\prime} \otimes \mathbf{q}^{\prime}}=-\mu_{t} \nabla \overline{\mathbf{v}}-\mu_{1 t} \nabla \overline{\mathbf{q}} \\
\mathbf{R}_{q L} \equiv K_{f} \overline{\mathbf{q}^{\prime} L^{\prime}}=K_{f t} \bar{q} \bar{L}
\end{gathered}
$$

In equation (3.3) we consider a modified Boussinesq's eddy viscosity assumption, namely $\nabla \overline{\mathbf{v}}$ and $\nabla \overline{\mathbf{q}}$ instead of $\nabla^{s} \overline{\mathbf{v}}$ and $\nabla^{s} \overline{\mathbf{q}}$ because the incompressible condition holds only for the velocity field and not for the heat flux. Thus, we might use the identity $2 \nabla \cdot \nabla^{s} \overline{\mathbf{v}}=\nabla \overline{\mathbf{v}}$ but not the identity $2 \nabla \cdot \nabla^{s} \overline{\mathbf{q}}=\nabla \overline{\mathbf{q}}$. Furthermore, the antisymmetric part of $\nabla \overline{\mathbf{v}}$ and $\nabla \overline{\mathbf{q}}$ could also play a role, besides that of the symmetric part, but it would be too cumbersome discussing it for the aim of the paper.

In (3.3)-(3.5), following the usual procedure of the zeroth order description of turbulence, we have introduced the turbulent parameters $\nu_{t}, \nu_{1 t}, \mu_{t}, \mu_{1 t}$, and $K_{f t}$, which, in analogy to the $K-\epsilon$ model of classical turbulence, will be phenomenologically expressed in terms of the second moments of $\mathbf{v}^{\prime}, \mathbf{q}^{\prime}$, which we write here as

$$
K \equiv \frac{1}{2} \overline{\mathbf{v}^{\prime} \cdot \mathbf{v}^{\prime}}, \quad K_{q} \equiv \frac{1}{2} \overline{\mathbf{q}^{\prime} \cdot \mathbf{q}^{\prime}}
$$

and of the corresponding dissipation functions $\epsilon$ and $\epsilon_{q}$ (see (3.13) and (3.21) below), related to the second moments of $\nabla \mathbf{v}^{\prime}$ and $\nabla \mathbf{q}^{\prime}$ appearing in the respective evolution equations of $K$ and $K_{q}$ (see (3.17) and (3.27)).

Equations (2.7) and (2.8) can be rewritten using (3.3)-(3.5). Thus, the equation for $\overline{\mathbf{v}}$ becomes

$$
\frac{\partial}{\partial t} \overline{\mathbf{v}}+(\overline{\mathbf{v}} \cdot \nabla) \overline{\mathbf{v}}+\frac{1}{\rho} \nabla \bar{p}-\left(\nu+\nu_{t}\right) \nabla^{2} \overline{\mathbf{v}}-\left(\nu_{1}+\nu_{1 t}\right) \nabla^{2} \overline{\mathbf{q}}=0
$$

and the equation for $\overline{\mathbf{q}}$ becomes

$$
\frac{\partial}{\partial t} \overline{\mathbf{q}}+(\overline{\mathbf{v}} \cdot \nabla) \overline{\mathbf{q}}+\zeta \nabla \bar{T}-\left(\mu+\mu_{t}\right) \nabla^{2} \overline{\mathbf{v}}-\left(\mu_{1}+\mu_{1 t}\right) \nabla^{2} \overline{\mathbf{q}}=-\left(K_{f}+K_{f t}\right) \bar{L} \overline{\mathbf{q}} .
$$

It is thus seen that the form of (3.3)-(3.5) has been chosen in such a way that the turbulent coefficients in them modify the molecular coefficients appearing in (2.7) and (2.8).

In analogy with (3.2), the coefficients $\nu_{t}, \nu_{1 t}, \mu_{t}, \mu_{1 t}$ and $K_{f t}$ will be expressed on dimensional grounds in terms of $K, \epsilon, K_{q}$ and $\epsilon_{q}$ taking into account that the dimensions of $K$ are

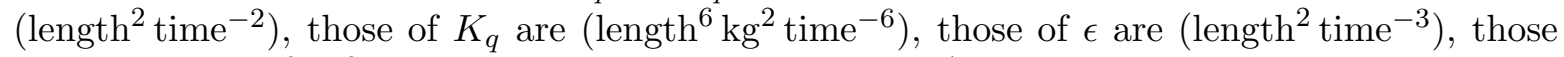
of $\epsilon_{q}$ are $\left(\right.$ length ${ }^{6} \mathrm{~kg}^{2}$ time $\left.^{-7}\right)$ and those of $\mu$ are $\left(\right.$ length ${ }^{4} \mathrm{~kg}$ time $\left.{ }^{-3}\right)$, those of $\nu_{t}, \mu_{1 t}$ and $K_{f t}$

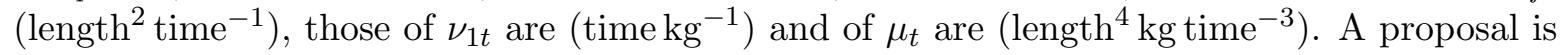

$$
\nu_{t}=C_{\nu} \frac{K^{2}}{\epsilon}, \quad \nu_{1 t}=\frac{C_{\nu 1}}{\rho s T} \frac{K^{2}}{\epsilon},
$$

and

$$
\mu_{t}=\frac{C_{\mu}}{\rho s T} \frac{K_{q}^{2}}{\epsilon_{q}}, \quad \mu_{1 t}=\frac{C_{\mu 1}}{(\rho s T)^{2}} \frac{K_{q}^{2}}{\epsilon_{q}}, \quad K_{f t}=\frac{C_{K}}{(\rho s T)^{2}} \frac{K_{q}^{2}}{\epsilon_{q}}
$$


with $C_{\nu}, C_{\nu 1}, C_{\mu}, C_{\mu 1}$ and $C_{K}$ dimensionless constants (to be found from the experiments), as it happens with the values of $C_{\nu}$ in the usual $K-\epsilon$ model. Of course, the turbulent parameters expressed above strongly depend on our choice, but different proposal would also be possible, as explained in the Conclusions.

Now, we need the evolution equation for the $K, \epsilon, K_{q}$ and $\epsilon_{q}$, which will give the evolution of (3.9) and (3.10). This will be done in Sections (3.2.2) and (3.2.3). An alternative possibility to $(3.9)$ and (3.10) will be discussed in the concluding remarks.

\subsubsection{Evolution equation and dissipation function for the second moment of $\mathrm{v}^{\prime}$}

The equation for the turbulent kinetic energy $K \equiv \frac{1}{2} \overline{\mathbf{v}^{\prime} \cdot \mathbf{v}^{\prime}}$ can be found writing the equation for $\mathbf{v}^{\prime}$ subtracting it from the equation $(2.4 \mathrm{~b})$, namely

$$
\frac{\partial}{\partial t} \mathbf{v}^{\prime}+\mathbf{v}^{\prime} \cdot \nabla \overline{\mathbf{v}}+\overline{\mathbf{v}} \cdot \nabla \mathbf{v}^{\prime}+\mathbf{v}^{\prime} \cdot \nabla \mathbf{v}^{\prime}+\frac{1}{\rho} \nabla p^{\prime}-\left(\nu \nabla^{2} \mathbf{v}^{\prime}+\nu_{1} \nabla^{2} \mathbf{q}^{\prime}\right)=0
$$

Multiplying this equation by $\mathbf{v}^{\prime}$ and averaging over the time we find the equation for $K$ :

$$
\frac{\partial}{\partial t} K+(\overline{\mathbf{v}} \cdot \nabla) K+\mathbf{R}_{v}: \nabla \overline{\mathbf{v}}+\nabla \cdot\left[\frac{1}{2} \overline{v^{\prime 2} \mathbf{v}^{\prime}}+\frac{1}{\rho} \overline{p^{\prime} \mathbf{v}^{\prime}}-\nu \overline{\mathbf{v}^{\prime} \cdot \nabla \mathbf{v}^{\prime}}-\nu_{1} \overline{\mathbf{v}^{\prime} \cdot \nabla \mathbf{q}^{\prime}}\right]=-\epsilon,
$$

with $\epsilon$, the dissipation function, given by

$$
\epsilon=\left[\nu \overline{\nabla \mathbf{v}^{\prime}: \nabla \mathbf{v}^{\prime}}+\nu_{1} \overline{\nabla \mathbf{v}^{\prime}: \nabla \mathbf{q}^{\prime}}\right] .
$$

The first term in (3.13) describes the usual viscous dissipation, but due to the fluctuating part of the turbulent viscosity field, and the second term describes the additional dissipation of kinetic energy related to the last term in equation (2.4a) namely to the interaction between velocity fluctuations and heat flux fluctuations.

Taking into account (3.3), we find

$\frac{\partial}{\partial t} K+(\overline{\mathbf{v}} \cdot \nabla) K+\nabla \cdot\left[\frac{1}{2} \overline{v^{\prime 2} \mathbf{v}^{\prime}}+\frac{1}{\rho} \overline{p^{\prime} \mathbf{v}^{\prime}}-\nu \overline{\mathbf{v}^{\prime} \cdot \nabla \mathbf{v}^{\prime}}-\nu_{1} \overline{\mathbf{v}^{\prime} \cdot \nabla \mathbf{q}^{\prime}}\right]=-\epsilon+\nu_{t} \nabla \overline{\mathbf{v}}: \nabla \overline{\mathbf{v}}+\nu_{1 t} \nabla \overline{\mathbf{v}}: \nabla \overline{\mathbf{q}}$

In analogy with what is usually made in classical turbulence, where the term in $\nabla K$ comes from the trace of $\mathbf{v}^{\prime} \cdot \nabla \mathbf{v}^{\prime}$. We propose the following further relation:

$$
-\nu \overline{\mathbf{v}^{\prime} \cdot \nabla \mathbf{v}^{\prime}}-\nu_{1} \overline{\mathbf{v}^{\prime} \cdot \nabla \mathbf{q}^{\prime}}=-\nu\left(1+\alpha_{1}\right) \nabla K
$$

with $\alpha_{1}$ a dimensionless constant, and

$$
\frac{1}{2} \overline{v^{\prime 2} \mathbf{v}^{\prime}}+\frac{1}{\rho} \overline{p^{\prime} \mathbf{v}^{\prime}}=-\frac{\nu_{t}^{*}}{\sigma_{K}} \nabla K
$$

where $\nu_{t}^{*}=\nu_{t}\left(1+\alpha_{1 t} \frac{C_{\nu 1}}{C_{\nu}}\right)$ with $\alpha_{1 t}$ and $\sigma_{K}$ numerical constants of the model. Thus, equation (3.14) becomes

$$
\frac{\partial}{\partial t} K+(\overline{\mathbf{v}} \cdot \nabla) K-\nabla \cdot\left[\left(\nu+\nu \alpha_{1}+\frac{\nu_{t}^{*}}{\sigma_{K}}\right) \nabla K\right]=-\epsilon+\frac{\nu_{t}}{\nu} \epsilon_{K}
$$


where $\epsilon_{K}=\nu \nabla \overline{\mathbf{v}}: \nabla \overline{\mathbf{v}}+\nu_{1} \nabla \overline{\mathbf{v}}: \nabla \overline{\mathbf{q}}$. Note that there is a relevant conceptual difference between $\epsilon$ defined in (3.13) and $\epsilon_{K}$ : the former one depends on the gradients of the fluctuating quantities $\mathbf{v}^{\prime}$ and $\mathbf{q}^{\prime}$, whereas the latter one depends on the gradients of the average values $\overline{\mathbf{v}}$ and $\overline{\mathbf{q}}$.

The ratio $\frac{K}{\epsilon}$ has the dimension of time and it is usually considered as a characteristic time for the decay of $\epsilon$. In analogy with what is done in the $K-\epsilon$ model [14], an evolution equation for $\epsilon$ can be heuristically obtained by keeping the same structure of the equation of $K$ and by dividing the right-hand side of equation (3.17) by the time $\frac{K}{\epsilon}$ yielding:

$$
\frac{\partial}{\partial t} \epsilon+(\overline{\mathbf{v}} \cdot \nabla) \epsilon-\nabla \cdot\left[\left(\nu+\nu \alpha_{1}+\frac{\nu_{t}^{*}}{\sigma_{\epsilon}}\right) \nabla \epsilon\right]=\frac{\epsilon}{K}\left[\frac{\nu_{t}}{\nu} C_{\epsilon 1} \epsilon_{K}-C_{\epsilon 2} \epsilon\right],
$$

with $C_{\epsilon 1}$ and $C_{\epsilon 2}$ numerical coefficients. In the $k-\epsilon$ model for viscous fluid $\left(\alpha_{1}=0\right), C_{\epsilon 1}=1.44$, $C_{\epsilon 2}=1.92, \sigma_{K}=1$ and $\sigma_{\epsilon}=1.4$ [14]. We tentatively take this equation for our proposal of the model. In fact, the form as that used in (3.18) has been justified in [14] on ground of analogy with kinetic theory using thermodynamics arguments.

\subsubsection{Evolution equation and dissipation function for the second moment of $q^{\prime}$}

Following for $\mathbf{q}$ the same procedure we have followed in subsection 3.2.2 to find the evolution equation for the second moments of $\mathbf{v}^{\prime}$, we write an equation for $\mathbf{q}^{\prime}$ :

$$
\frac{\partial}{\partial t} \mathbf{q}^{\prime}+\mathbf{v}^{\prime} \cdot \nabla \overline{\mathbf{q}}+\overline{\mathbf{v}} \cdot \nabla \mathbf{q}^{\prime}+\mathbf{v}^{\prime} \cdot \nabla \mathbf{q}^{\prime}+\zeta \nabla T^{\prime}-\mu \nabla^{2} \mathbf{v}^{\prime}-\mu_{1} \nabla^{2} \mathbf{q}^{\prime}+K_{f}\left(\bar{L} \mathbf{q}^{\prime}+L^{\prime} \overline{\mathbf{q}}+L^{\prime} \mathbf{q}^{\prime}-\overline{L^{\prime} \mathbf{q}^{\prime}}\right)-\overline{\mathbf{v}^{\prime} \cdot \nabla \mathbf{q}^{\prime}}=0
$$

We multiply (3.19) times $\mathbf{q}^{\prime}$ and after averaging over the time we find the equation for the second moments of $\mathbf{q}^{\prime}$, expressed as $K_{q}=\frac{1}{2} \overline{\mathbf{q}^{\prime} \cdot \mathbf{q}^{\prime}}$ :

$$
\begin{gathered}
\frac{\partial}{\partial t} K_{q}+(\overline{\mathbf{v}} \cdot \nabla) K_{q}+\overline{\mathbf{q}^{\prime} \cdot\left(\mathbf{v}^{\prime} \cdot \nabla\right) \overline{\mathbf{q}}}-\zeta \overline{T^{\prime} \nabla \cdot \mathbf{q}^{\prime}}+\nabla \cdot\left[\zeta \overline{T^{\prime} \mathbf{q}^{\prime}}-\mu \overline{\nabla \mathbf{v}^{\prime} \cdot \mathbf{q}^{\prime}}-\mu_{1} \overline{\mathbf{q}^{\prime} \cdot \nabla \mathbf{q}^{\prime}}\right]= \\
=-\epsilon_{q}-\overline{\mathbf{q}^{\prime} \cdot\left(\mathbf{v}^{\prime} \cdot \nabla \mathbf{q}^{\prime}\right)}-K_{f}\left(2 \bar{L} K_{q}+\overline{L^{\prime} \overline{\mathbf{q}} \cdot \mathbf{q}^{\prime}}+\overline{L^{\prime} \mathbf{q}^{\prime} \cdot \mathbf{q}^{\prime}}\right),
\end{gathered}
$$

where we have used the identity $\mathbf{q}^{\prime} \cdot \nabla^{2} \mathbf{v}^{\prime}=\mathbf{q}^{\prime} \cdot\left(\nabla \cdot \nabla \mathbf{v}^{\prime}\right)=\nabla \cdot\left(\nabla \mathbf{v}^{\prime} \cdot \mathbf{q}^{\prime}\right)-\nabla \mathbf{v}^{\prime}: \nabla \mathbf{q}^{\prime}$, and $\mathbf{q}^{\prime} \cdot \nabla^{2} \mathbf{q}^{\prime}=\mathbf{q}^{\prime} \cdot\left(\nabla \cdot \nabla \mathbf{q}^{\prime}\right)=\nabla \cdot\left(\nabla \mathbf{q}^{\prime} \cdot \mathbf{q}^{\prime}\right)-\nabla \mathbf{q}^{\prime}: \nabla \mathbf{q}^{\prime}$ and we have defined the dissipation function $\epsilon_{q}$ for $K_{q}$ as

$$
\epsilon_{q}=\left[\mu \overline{\nabla \mathbf{v}^{\prime}: \nabla \mathbf{q}^{\prime}}+\mu_{1} \overline{\nabla \mathbf{q}^{\prime}: \nabla \mathbf{q}^{\prime}}\right],
$$

Equation (3.20) can be also written

$$
\begin{aligned}
\frac{\partial}{\partial t} K_{q}+ & (\overline{\mathbf{v}} \cdot \nabla) K_{q}+\nabla \cdot\left[\overline{\mathbf{v}^{\prime} \frac{q^{\prime 2}}{2}}+\zeta \overline{T^{\prime} \mathbf{q}^{\prime}}-\mu \overline{\nabla \mathbf{v}^{\prime} \cdot \mathbf{q}^{\prime}}-\mu_{1} \overline{\mathbf{q}^{\prime} \cdot \nabla \mathbf{q}^{\prime}}\right]= \\
& =-\epsilon_{q}-\mathbf{R}_{q}: \nabla \overline{\mathbf{q}}+\zeta \overline{T^{\prime} \nabla \cdot \mathbf{q}^{\prime}}-K_{f}\left(2 \bar{L} K_{q}+\overline{L^{\prime} \overline{\mathbf{q}} \cdot \mathbf{q}^{\prime}}\right)
\end{aligned}
$$

where we have used $\mathbf{q}^{\prime} \cdot\left(\mathbf{v}^{\prime} \cdot \nabla \mathbf{q}^{\prime}\right)=\nabla \cdot\left(\mathbf{v}^{\prime} \frac{q^{\prime 2}}{2}\right)$ and neglected the third-order moment $\overline{L^{\prime} \mathbf{q}^{\prime} \cdot \mathbf{q}^{\prime}}$.

In analogy with (3.15), we propose the constitutive relations: 


$$
-\mu \overline{\nabla \mathbf{v}^{\prime} \cdot \mathbf{q}^{\prime}}-\mu_{1} \overline{\mathbf{q}^{\prime} \cdot \nabla \mathbf{q}^{\prime}}=-\mu\left(1+\alpha_{2}\right) \nabla K_{q}
$$

with $\alpha_{2}$ a dimensionless constant and in analogy with (3.16)

$$
\frac{1}{2} \overline{\mathbf{v}^{\prime} q^{\prime 2}}+\zeta \overline{T^{\prime} \mathbf{q}^{\prime}}=-\frac{\mu_{t}^{*}}{\sigma_{q}} \nabla K_{q}
$$

where $\mu_{t}^{*}=\mu_{t}\left(1+\alpha_{2 t} \frac{C_{\mu 1}}{C_{\mu}}\right)$ with $\alpha_{2 t}$ and $\sigma_{q}$ numerical constants of the model, analogous to $\alpha_{1 t}$ and $\sigma_{K}$ in (3.16).

Equation (3.14) becomes

$\frac{\partial}{\partial t} K_{q}+(\overline{\mathbf{v}} \cdot \nabla) K_{q}-\nabla \cdot\left[\left(\mu+\mu \alpha_{2}+\frac{\mu_{t}^{*}}{\sigma_{K q}}\right) \nabla K_{q}\right]=-\epsilon_{q}+\frac{\mu_{t}}{\mu} \epsilon_{K q}+\zeta \overline{T^{\prime} \nabla \cdot \mathbf{q}^{\prime}}-2 K_{f} \bar{L} K_{q}-K_{f t} \bar{q}^{2} \bar{L}$,

where $\epsilon_{K q}=\mu \nabla \overline{\mathbf{v}}: \nabla \overline{\mathbf{q}}+\mu_{1} \nabla \overline{\mathbf{q}}: \nabla \overline{\mathbf{q}}$. Note that as well as $\epsilon_{K}$, the quantity $\epsilon_{K q}$ depends on the gradients of the average values $\overline{\mathbf{v}}$ and $\overline{\mathbf{q}}$ in contrast to $\epsilon_{q}$ in (3.21), which depends on the fluctuating part of $\overline{\mathbf{v}}$ and $\overline{\mathbf{q}}$.

For the terms $\zeta \overline{T^{\prime} \nabla \cdot \mathbf{q}^{\prime}}$ and $2 K_{f} 2 \bar{L} K_{q}+K_{f t} \bar{q}^{2} \bar{L}$ in (3.25) we propose

$$
\zeta \overline{T^{\prime} \nabla \cdot \mathbf{q}^{\prime}}-2 K_{f} \bar{L} K_{q}-K_{f t} \bar{q}^{2} \bar{L} \equiv-2 K_{f}^{*} \bar{L} K_{q}
$$

in such a form that $K_{f}^{*}$ would contribute to modify the mutual friction coefficient $K_{f}$ of equation (3.25). Inserting the costitutive relation (3.26) in (3.25), we find:

$$
\frac{\partial}{\partial t} K_{q}+(\overline{\mathbf{v}} \cdot \nabla) K_{q}-\nabla \cdot\left[\left(\mu+\mu \alpha_{2}+\frac{\mu_{t}^{*}}{\sigma_{K q}}\right) \nabla K_{q}\right]=-\epsilon_{q}+\frac{\mu_{t}}{\mu} \epsilon_{K q}-2\left(K_{f}+K_{f}^{*}\right) \bar{L} K_{q}
$$

We can also find an evolution equation for $\epsilon_{q}$ following the same procedure used in (3.18), namely by keeping the same structure of Eq. (3.27) for the left-hand side and multiplying the right hand side of Eq. (3.27) with the reciprocal of the characteristic time $\frac{\epsilon_{q}}{K_{q}}$ :

$\frac{\partial}{\partial t} \epsilon_{q}+(\overline{\mathbf{v}} \cdot \nabla) \epsilon_{q}-\nabla \cdot\left[\left(\mu+\mu \alpha_{2}+\frac{\mu_{t}^{*}}{\sigma_{\epsilon q}}\right) \nabla \epsilon_{q}\right]=\frac{\epsilon_{q}}{K_{q}}\left[-C_{\epsilon 2 q} \epsilon_{q}+C_{\epsilon 1 q} \epsilon_{K q}-2 C_{\epsilon 3 q}\left(K_{f}+K_{f}^{*}\right) \bar{L} K_{q}\right]$.

with $C_{\epsilon 2 q}, C_{\epsilon 1 q}$ and $C_{\epsilon 3 q}$ numerical constants. This equation plays an analogous role than the evolution equations for $\epsilon$ derived in (3.18). We take as an explicit illustration of our proposal, but the method could be open to more general evolution equations for $\epsilon_{q}$.

\section{Evolution equation and dissipation function for $\bar{L}$}

After having considered a closure of equations (2.7) and (2.8) for $\overline{\mathbf{v}}$ and $\overline{\mathbf{q}}$, we consider the fluctuations in the evolution equation for $\bar{L}(2.11)$, namely $\mathbf{R}_{v L} \equiv \overline{\mathbf{v}^{\prime} L^{\prime}}, \mathbf{R}_{q \mathcal{L}} \equiv \overline{\mathbf{q}^{\prime} \mathcal{L}^{\prime}}, \mathbf{R}_{\mathcal{L} \mathcal{L}} \equiv$ $\overline{\mathcal{L}^{\prime} \mathcal{L}^{\prime}}, \mathbf{R}_{\mathcal{L L L}} \equiv \overline{\mathcal{L}^{\prime} \mathcal{L}^{\prime} \mathcal{L}^{\prime}}, \mathbf{R}_{\mathcal{L} \mathcal{L} \mathcal{L} \mathcal{L}} \equiv \overline{\mathcal{L}^{\prime} \mathcal{L}^{\prime} \mathcal{L}^{\prime} \mathcal{L}^{\prime}}, \mathbf{R}_{q \mathcal{L L}} \equiv \overline{q^{\prime} \mathcal{L}^{\prime} \mathcal{L}^{\prime}}$ and $\mathbf{R}_{q \mathcal{L} \mathcal{L} \mathcal{L}} \equiv \overline{q^{\prime} \mathcal{L}^{\prime} \mathcal{L}^{\prime} \mathcal{L}^{\prime}}$. To keep the form of equation (2.9) we propose the following expressions to close equation (2.11):

$$
\mathbf{R}_{v L} \equiv \overline{\mathbf{v}^{\prime} L^{\prime}}=-D_{L t} \nabla L
$$




$$
\begin{aligned}
& \mathbf{R}_{q \mathcal{L}} \equiv \overline{q^{\prime} \mathcal{L}^{\prime}}=\alpha_{t} \bar{q} \overline{\mathcal{L}} \\
& \mathbf{R}_{\mathcal{L L}} \equiv \overline{\mathcal{L}^{\prime} \mathcal{L}^{\prime}}=\beta_{t} \overline{\mathcal{L}}^{2}
\end{aligned}
$$

Closure in equation (4.29) follows the same strategy used in the paper, namely equation for $\bar{L}$ has the same form as that for $L$, but with effective diffusion parameter.

For the third and fourth moments we assume that the fluctuations in $\mathcal{L}^{\prime}$ are Gaussian, namely $\mathbf{R}_{\mathcal{L L L}} \equiv{\overline{\mathcal{L}^{\prime}}}^{3}=0, \mathbf{R}_{\mathcal{L L L L}} \equiv{\overline{\mathcal{L}^{\prime}}}^{4}=3 \mathbf{R}_{\mathcal{L L} \mathcal{L}}^{2}$, and that $\mathbf{R}_{q \mathcal{L L}} \equiv{\overline{q^{\prime} \mathcal{L}^{\prime}}}^{2}=0$ and $\mathbf{R}_{q \mathcal{L L L}} \equiv$ ${\overline{q^{\prime} \mathcal{L}^{\prime}}}^{3}=0$.

With the assumptions (4.29)-(4.31) and introducing them into (2.11), it is seen that $\mathbf{R}_{v L}$ modifies the effective diffusion coefficient $D_{L}$ of the quantized vortices with the turbulent contribution $D_{L t}$, while $\mathbf{R}_{q L}$ contributes to the production of quantized vortices and $\mathbf{R}_{L L}$ contributes both to the production terms and the destruction term.

In (4.29)-(4.31), we have introduced the phenomenological turbulent parameters $D_{L t}, \alpha_{t}$ and $\beta_{t}$, which will be expressed in terms of the second moments of $L$, namely

$$
K_{L} \equiv \frac{1}{2} \overline{L^{\prime} \cdot L^{\prime}}
$$

and on the corresponding dissipation function $\epsilon_{L}$ (see (4.37) below) in the equation of $K_{L}$ (see (4.42)) the turbulent diffusion coefficient will be

$$
D_{L t}=C_{L} \kappa^{2} \ell^{2} \frac{K_{L}^{2}}{\epsilon_{L}}
$$

with $C_{L}$ a dimensionless constant, and the quantities $\alpha_{t}$ and $\beta_{t}$ in (4.30) and (4.31) are dimensionless constants.

The equation (2.11) of the time-averaged vortex line density becomes

$$
\frac{\partial}{\partial t} \bar{L}+\overline{\mathbf{v}} \cdot \nabla \bar{L}-\left(D_{L}+D_{L t}\right) \nabla^{2} \bar{L}=\tilde{\alpha} \bar{q} \overline{\mathcal{L}}^{3}-\tilde{\beta} \kappa \overline{\mathcal{L}}^{4}
$$

with $\tilde{\alpha}=\alpha\left(1+3 \beta_{t}+3 \alpha_{t}\right)$ and $\tilde{\beta}=\beta\left(1+9 \beta_{t}\right)$, which can be also written in terms of $\bar{L}$ as

$$
\frac{\partial}{\partial t} \bar{L}+\overline{\mathbf{v}} \cdot \nabla \bar{L}-\left(D_{L}+D_{L t}\right) \nabla^{2} \bar{L}=\tilde{\alpha} \bar{q} \bar{L}^{3 / 2}-\tilde{\beta} \kappa \bar{L}^{2}
$$

Now, we follow the same procedure of the two former subsections for the equation of the vortex line density (2.4c). Thus, let's find the equation for $L^{\prime}$ :

$$
\begin{gathered}
\frac{\partial}{\partial t} L^{\prime}+\overline{\mathbf{v}} \cdot \nabla L^{\prime}+\mathbf{v}^{\prime} \cdot \nabla \bar{L}+\mathbf{v}^{\prime} \cdot \nabla L^{\prime}-\overline{\mathbf{v}^{\prime} \cdot \nabla L^{\prime}}-D_{L} \nabla^{2} L^{\prime}= \\
+\alpha \bar{q}\left(3 \overline{\mathcal{L}}^{2} \mathcal{L}^{\prime}+3 \overline{\mathcal{L}} \mathcal{L}^{\prime 2}+\mathcal{L}^{\prime 3}\right)-\beta \kappa\left(4 \overline{\mathcal{L}}^{3} \mathcal{L}^{\prime}+6 \overline{\mathcal{L}}^{2} \mathcal{L}^{\prime 2}+4 \overline{\mathcal{L}} \mathcal{L}^{\prime 3}+\mathcal{L}^{\prime 4}\right)+ \\
\alpha q^{\prime}\left(\overline{\mathcal{L}}^{3}+3 \overline{\mathcal{L}}^{2} \mathcal{L}^{\prime}+3 \overline{\mathcal{L}} \mathcal{L}^{\prime 2}+\mathcal{L}^{\prime 3}\right)-3 \alpha \bar{q} \overline{\mathcal{L}} \overline{\mathcal{L}^{\prime 2}}+\beta \kappa\left(6 \overline{\mathcal{L}^{2}} \overline{\mathcal{L}^{\prime 2}}+\overline{\mathcal{L}^{\prime 4}}\right)-\alpha\left(3 \overline{\mathcal{L}}^{2} \overline{q^{\prime} \mathcal{L}^{\prime}}+3 \overline{\mathcal{L}} \overline{q^{\prime} \mathcal{L}^{\prime 2}}\right)
\end{gathered}
$$

Let's multiply equation (4.36) by $L^{\prime}$ yielding the equation for $K_{L}=\frac{1}{2} \overline{L^{\prime} L^{\prime}}$ :

$$
\frac{\partial}{\partial t} K_{L}+\overline{\mathbf{v}} \cdot \nabla K_{L}+\nabla \cdot\left[\overline{\mathbf{v}^{\prime} \frac{L^{\prime 2}}{2}}-D_{L} \nabla K_{L}\right]=-\epsilon_{L}-\mathbf{R}_{v L} \cdot \nabla \bar{L}+
$$




$$
\begin{gathered}
+\alpha \bar{q}\left(3 \overline{\mathcal{L}^{2}} \overline{L^{\prime} \mathcal{L}^{\prime}}+3 \overline{\mathcal{L}} \overline{L^{\prime} \mathcal{L}^{\prime 2}}+\overline{L^{\prime} \mathcal{L}^{\prime 3}}\right)-\beta \kappa\left(4 \overline{\mathcal{L}^{3}} \overline{L^{\prime} \mathcal{L}^{\prime}}+6 \overline{\mathcal{L}^{2}} \overline{L^{\prime} \mathcal{L}^{\prime 2}}+4 \overline{\mathcal{L}} \overline{L^{\prime} \mathcal{L}^{\prime 3}}+\overline{L^{\prime} \mathcal{L}^{\prime 4}}\right)+ \\
+\alpha\left(\overline{\mathcal{L}^{3}} \overline{q^{\prime} L^{\prime}}+3 \overline{\mathcal{L}^{2}} \overline{q^{\prime} L^{\prime} \mathcal{L}^{\prime}}+3 \overline{\mathcal{L}} \overline{q^{\prime} L^{\prime} \mathcal{L}^{\prime 2}}+\overline{q^{\prime} L^{\prime} \mathcal{L}^{\prime 3}}\right)
\end{gathered}
$$

where we have used $L^{\prime} \overline{\mathbf{v}} \cdot \nabla L^{\prime}=\overline{\mathbf{v}} \cdot \nabla K_{L}$, and $\mathbf{R}_{v L}=\overline{\mathbf{v}^{\prime} L^{\prime}}=-D_{L t} \nabla \bar{L}$, as defined in (4.29). In (4.37) the dissipation function $\epsilon_{L}$ is given by

$$
\epsilon_{L} \equiv D_{L} \overline{\nabla L^{\prime} \cdot \nabla L^{\prime}}
$$

This plays an analogous role to $\epsilon$ (3.13) and $\epsilon_{q}$ (3.21) in the equation for $K$ and for $K_{q}$, respectively.

In the equation (4.37) we assume that

$$
\overline{\mathbf{v}^{\prime} \frac{L^{\prime 2}}{2}} \simeq-\frac{D_{L t}}{\sigma_{K L}} \nabla K_{L}
$$

with $D_{L t}$ given in (4.33). Furthermore, we assume for the perturbations on the right-hand side of equation (4.37) that

$$
\begin{gathered}
\alpha \bar{q}\left(3 \overline{\mathcal{L}}^{2} \overline{L^{\prime} \mathcal{L}^{\prime}}+3 \overline{\mathcal{L}} \overline{L^{\prime} \mathcal{L}^{\prime 2}}+\overline{L^{\prime} \mathcal{L}^{\prime 3}}\right)+\alpha\left(\overline{\mathcal{L}}^{3} \overline{q^{\prime} L^{\prime}}+3 \overline{\mathcal{L}^{2}} \overline{q^{\prime} L^{\prime} \mathcal{L}^{\prime}}+3 \overline{\mathcal{L}} \overline{q^{\prime} L^{\prime} \mathcal{L}^{\prime 2}}+\overline{q^{\prime} L^{\prime} \mathcal{L}^{\prime 3}}\right) \simeq \\
\simeq 3 \alpha \bar{q} \overline{\mathcal{L}}^{2} \overline{L^{\prime} \mathcal{L}^{\prime}}+\alpha \overline{\mathcal{L}}^{3} \overline{q^{\prime} L^{\prime}} \simeq 3 \alpha \beta_{t} \bar{q} \overline{\mathcal{L}}^{3} \bar{L}+\alpha \alpha_{t} \bar{q} \overline{\mathcal{L}}^{3} \bar{L}=\left(3 \alpha \beta_{t}+\alpha \alpha_{t}\right) \bar{L} \bar{q} \overline{\mathcal{L}}^{3}=\left(3 \alpha \beta_{t}+\alpha \alpha_{t}\right) \bar{q} \bar{L}^{5 / 2}
\end{gathered}
$$

and that

$$
\beta \kappa\left(4 \overline{\mathcal{L}}^{3} \overline{L^{\prime} \mathcal{L}^{\prime}}+6 \overline{\mathcal{L}}^{2} \overline{L^{\prime} \mathcal{L}^{\prime 2}}+4 \overline{\mathcal{L}} \overline{L^{\prime} \mathcal{L}^{\prime 3}}+\overline{L^{\prime} \mathcal{L}^{\prime 4}}\right) \simeq 4 \beta \kappa \overline{\mathcal{L}}^{3} \overline{L^{\prime} \mathcal{L}^{\prime}} \simeq 4 \beta \beta_{t} \kappa \bar{L} \overline{\mathcal{L}}^{4}=4 \beta \beta_{t} \kappa \bar{L}^{3}
$$

because of $\mathcal{L}^{1 / 2}=L$.

Equation (4.37) thus becomes:

$\frac{\partial}{\partial t} K_{L}+\overline{\mathbf{v}} \cdot \nabla K_{L}-\nabla \cdot\left[\left(D_{L}+\frac{D_{L t}}{\sigma_{K L}}\right) \nabla K_{L}\right]=-\epsilon_{L}+\frac{D_{L t}}{D_{L}} \epsilon_{K L}+\left(3 \alpha \beta_{t}+\alpha \alpha_{t}\right) \bar{q} \bar{L}^{5 / 2}-4 \beta \beta_{t} \kappa \bar{L}^{3}$

where $\epsilon_{K L}=D_{L} \nabla \bar{L} \cdot \nabla \bar{L}$, which depends on the gradients of the average value $\bar{L}$.

As in (3.18) and in (3.28) an evolution equation for $\epsilon_{L}$ is found keeping for the left-hand side a structure analogous to that of (4.42), and the right-hand side if found by multiplying the right-hand side of equation (4.42) by the characteristic time $\frac{\epsilon_{L}}{K_{L}}$ :

$$
\begin{gathered}
\frac{\partial}{\partial t} \epsilon_{L}+\overline{\mathbf{v}} \cdot \nabla \epsilon_{L}-\nabla \cdot\left[\left(D_{L}+\frac{D_{L t}}{\sigma_{\epsilon L}}\right) \nabla \epsilon_{L}\right]= \\
=\frac{\epsilon_{L}}{K_{L}}\left(-C_{L 1} \epsilon_{L}+C_{L 2} \frac{D_{L t}}{D_{L}} \epsilon_{K L}+C_{L 3}\left(3 \alpha \beta_{t}+\alpha \alpha_{t}\right) \bar{q} \bar{L}^{5 / 2}-4 C_{L 4} \beta \beta_{t} \kappa \bar{L}^{3}\right)
\end{gathered}
$$

with $C_{L i}$ dimensionless constants.

A simple illustration would be counterflow transport with turbulent fluctuations of the heat flux q. Equation (2.4b) has been considered in detail in the laminar regime $(L=0)$ and in quantum turbulent regime $(L \neq 0)$. In such analyses, the turbulent effects were focused 
on $L$, and it was assumed that $\mathbf{q}$ itself was not fluctuating. In this case, the average of the right-hand term in $(2.4 \mathrm{~b})$, namely $K_{f} L \mathbf{q}$, was simply $K_{f} \bar{L} \overline{\mathbf{q}}$, because $\mathbf{q}$ could be identified with its average value $\overline{\mathbf{q}}$. Thus, the fluctuations of $L$ itself disappeared from this term.

However, when $\mathbf{q}$ itself becomes a fluctuating quantity, the term $K_{f} L \mathbf{q}$ will be $K_{f}(\bar{L}+$ $\left.L^{\prime}\right)\left(\overline{\mathbf{q}}+\mathbf{q}^{\prime}\right)$, and its average will be $K_{f}\left(\bar{L} \overline{\mathbf{q}}+\overline{L^{\prime} \mathbf{q}^{\prime}}\right)$. In this case, the proposal (3.5) would be writing $K_{f} \overline{L^{\prime} \mathbf{q}^{\prime}}$ as $K_{f t} L \overline{\mathbf{q}}$, with $K_{f t}$ turbulent contribution to an effective friction coefficient. Thus, for $\mathbf{v}=0$ (for the sake of simplicity), equation (2.4b) for the heat flux would lead to (see $(2.8))$

$$
\frac{\partial}{\partial t} \overline{\mathbf{q}}+\zeta \nabla \bar{T}-\mu_{1} \nabla^{2} \overline{\mathbf{q}}=-\left(K_{f}+K_{f t}\right) \bar{L} \overline{\mathbf{q}}
$$

with $K_{f t}$ being a fluctuation of $K_{q}, \epsilon_{q}$, and $K_{L}$ and $\epsilon_{L}$ defined in (3.21), (4.32) and (4.38). The coefficient $K_{f t}$ would thus show the effects of the fluctuations of $L$ (which is a very relevant physical quantity, because $L$ may be strongly fluctuating, on the dynamics of $\overline{\mathbf{q}}$ ). In fact, it could be that $K_{f t}$ depended itself on the quantum Reynolds number $R e_{q}=\bar{q} d /(S T \kappa)$. If it depended as $K_{f t} \simeq\left(R_{q}-R_{q c}\right)^{\alpha}$, with $R_{q c}$ a critical Reynolds number for the fluctuations of $\mathbf{q}$ itself, one would have on the right-hand side a contribution of the form $\mathbf{q}^{3+a}$ (for $R e_{q}$ sufficiently higher than $\left.R e_{q c}\right)$. Indeed, some authors have reported that in some occasions, instead, the Gorter-Mellinck law $\nabla T \sim \mathbf{q}^{3}$, coming from $L \sim \mathbf{q}^{2}$ in the steady-state, and $K_{f}$ not dependent on $\mathbf{q}$, one observes $\nabla T \sim \mathbf{q}^{3.4}$. This interpretation for the origin of $\mathbf{q}^{3.4}$ is speculative, but we mention it as a concrete possibility of the coupled effects of fluctuations in $L$ and $\mathbf{q}$. If $\mathbf{v}$ is also fluctuating around $\mathbf{v}=0$, the terms in $\mathbf{v}^{\prime} \mathbf{q}^{\prime}$ will also play a role (see equation $(2.8))$.

\section{Discussion and concluding remarks}

In this paper we propose a generalized $K-\epsilon$ model applied to superfluid helium. Because of the fields involved in it, we have named this model $K-\epsilon-L$ model, where $L$ is the vortex length per unit volume. The starting points are the dynamical equations of the one-fluid extended model (2.4) for $\mathbf{v}, \mathbf{q}$ and $L$ [18]. Thus, it is natural that second moments of the fluctuations and second moments of the gradient of the fluctuations of $\mathbf{v}, \mathbf{q}$ and $L$ appear in this model, namely $K, K_{q}, K_{L}, \epsilon, \epsilon_{q}$ and $\epsilon_{L}$.

The complete $K-\epsilon-L$ model is thus given by

- the evolution equations for the averaged fields, namely (3.7) for $\overline{\mathbf{v}},(3.8)$ for $\overline{\mathbf{q}}$ and (4.34) for $\bar{L}$, together with $\nabla \cdot \overline{\mathbf{v}}=0$;

- the evolution equations for the second moments of the fluctuations and of their gradients, namely (3.17) for $K,(3.18)$ for $\epsilon,(3.27)$ for $K_{q},(3.28)$ for $\epsilon_{q},(4.42)$ for $K_{L}$ and (4.43) for $\epsilon_{L}$;

- the expressions for the phenomenological turbulent coefficients used in the closure, namely (3.9) for $\nu_{t}, \nu_{1 t},(3.10)$ for $\mu_{t}, \mu_{1 t}$ and $K_{f t}$ and (4.33) for $D_{L t}$ in terms of $K, \epsilon, K_{q}, \epsilon_{q}$, $K_{L}$, and $\epsilon_{L}$.

- The numerical constants appearing in the evolution equation (3.17) and (3.18) for $K$ and $\epsilon,(3.27)$ and (3.28) for $K_{q}$ and $\epsilon_{q}$, and (4.42) and (4.43) for $K_{L}$ and $\epsilon_{L}$. Those involving $K$ and $\epsilon$ are known from the usual viscous fluids [14]. 
(3.9), (3.10) and (4.33) as well as $\sigma_{K}$ in (3.16), $C_{\nu}$ and $C_{\nu 1}$ in (3.9), and $\sigma_{q}$ in (3.24), $C_{\nu}$ and $C_{\nu 1}$ in (3.10), $\sigma_{K L}$ in (4.39), and $C_{L}$ in (4.33).

This model is an open proposal rather than a definite one. Expressions (3.9), (3.10) and (4.33) for the turbulent coefficients in terms of $K, K_{q}$, and $K_{L}$, and $\epsilon, \epsilon_{q}$ and $\epsilon_{L}$ could be further discussed as well as the equations (3.18), (3.27) and (4.43) for the evolution of $\epsilon, \epsilon_{q}$ and $\epsilon_{L}$. For instance, concerning expressions (3.9) and (3.10) one could argue that since the terms in $\nu_{t}, \nu_{1 t}, \mu_{t}, \mu_{1 t}$ in (3.3) and in (3.4) couple $\mathbf{v}$ and $\mathbf{q}$, an alternative proposal for $\nu_{1 t}$ in (3.9) and for $\mu_{t}$ in (3.10) in terms if $K, \epsilon, K_{q}$ and $\epsilon_{q}$ could be

$$
\nu_{1 t}=\frac{C_{\nu 1}}{\sqrt{\rho s T}} \frac{K K_{q}}{\sqrt{\epsilon \epsilon_{q}}}, \quad \mu_{t}=\frac{C_{\mu}^{\prime}}{\sqrt{\rho s T}} \frac{K K_{q}}{\sqrt{\epsilon \epsilon_{q}}} .
$$

Of course, these equations strongly depend on the approximations made in the above sections, but they have been suggested by the structure of the equations themselves and keeping in mind the main procedure used for the classical turbulence. Indeed, some higher moments contribute to change the effective viscosity of the equation, or the diffusion coefficient or the production/destruction coefficients in their correspective equations.

Furthermore, the fluctuations of $L$ are especially complex and they depend on the kind of flow. The studies performed by Nemirovskii in Ref. [39] have shown that different kinds of turbulence (uniformly distributed or bundles of quantized vortices) lead to different expression for the spectrum of $L$. Thus, a simple proposal as the one made in this paper is not expected to cover all the complexities of the problem, but may provide a useful starting point for a phenomenological modelization. For these reasons, we have chosen the Vinen equation for $L$ with an inertial term, as the simplest model for describing turbulence in a uniformly distributed turbulence. In future analyses, more complex situations should be analyzed. Incidentally, Eq. (4.44) could provide a strategy for measuring different contributions of the fluctuations of $L$ if one makes $\mathbf{q}$ to fluctuate in a sinusoidal way with frequencies comparable to the range of frequencies of fluctuations of $L$, instead of letting q fluctuating in a turbulent way.

Two important issues in this paper are: a) the appearance of further kinds of "viscosity" (thermal and classical), which do not have analogous in other systems, as well as the appearance of their respective "turbulent" contributions, which open new viewpoints on viscosity and turbulence; b) the extension of the $K-\epsilon$ model of usual viscous systems to physical systems with higher freedom degrees.

In this paper we have proposed the $K-\epsilon-L$ model using the one-fluid extended model, based on the Extended Thermodynamics. The so-called two-fluid model proposed by Tisza and Landau $[15,16]$, sees superfluid helium as composed by two undistinguishable components: the normal component (viscous fluid which carries all the entropy and heat of helium) and the superfluid component (unviscid fluid). This is the model mostly used for studying superfluid helium, which has allowed to explain some properties of Helium II observed in the experiments (see Ref. $[12,53,54]$ for more details), as for instance the propagation of the second sound. A comparison between the two existing models can be seen in $[18,55]$. In that model, equation (2.4) of our model would be replaced by evolution equations for $\mathbf{v}_{n}$ and $\mathbf{v}_{s}$, the respective velocities of the normal and superfluid component, namely, the HVBK equations [12, 18]. The relations between $\mathbf{v}_{n}$ and $\mathbf{v}_{s}$, and $\mathbf{v}$ and $\mathbf{q}$ are $\mathbf{v}=\rho_{n} \mathbf{v}_{n}+\rho_{s} \mathbf{v}_{s}, \mathbf{q}=\rho_{s} T s\left(\mathbf{v}_{n}-\mathbf{v}_{s}\right)$, with $\rho_{n}$ and $\rho_{s}$ the mass density of the normal and superfluid components. A procedure analogous to that used here, namely, writing $\mathbf{v}_{n}=\overline{\mathbf{v}}_{n}+\mathbf{v}_{n}^{\prime}$ and $\mathbf{v}_{s}=\overline{\mathbf{v}}_{s}+\mathbf{v}_{s}^{\prime}$ could be used. 
Terms in $\overline{\mathbf{v}_{n}^{\prime} \otimes \mathbf{v}_{n}^{\prime}}, \overline{\mathbf{v}_{s}^{\prime} \otimes \mathbf{v}_{s}^{\prime}}$ and $\overline{\mathbf{v}_{n}^{\prime} \otimes \mathbf{v}_{s}^{\prime}}$ will appear in the time-average equations for $\overline{\mathbf{v}}_{n}$ and $\overline{\mathbf{v}}_{s}$. To close the equations, such second moments should be expressed in terms of $\nabla \overline{\mathbf{v}}_{n}$ and $\nabla \overline{\mathbf{v}}_{s}$, as in (3.3) and (3.4) - and also for coupling $\mathbf{v}_{n}^{\prime}, \mathbf{v}_{s}^{\prime}$ and $L^{\prime}$. - Thus, the ideas presented in this paper could also be explored in the context of the two-fluid model.

\section{Acknowledgements}

D.J. acknowledges the financial support from the Dirección General de Investigación of the Spanish Ministry of Economy and Competitiveness under grant RTI2018-097876-B-C22 and of the Direcció General de Recerca of the Generalitat of Catalonia, under grant 2017SGR1018. M.S. acknowledges the financial support of the Istituto Nazionale di Alta Matematica (GNFMGruppo Nazionale della Fisica Matematica), of the Università di Palermo (Contributo Cori 2017 - Azione D) and the hospitality of the "Group of Fisica Estadistica of the Universitat Autònoma de Barcelona".

\section{References}

[1] G. Ahmadi, "On thermodynamics of turbulence," Bulletin of the American Physical Society, vol. 29, p. 1529, 1984.

[2] N. Andersson, T. Sidery, and G. L. Comer, "Superfluid neutron star turbulence," Monthly Notices of the Royal Astronomical Society, vol. 381, pp. 747-756, 2007.

[3] C. F. Barenghi, R. J. Donnelly, and W. F.Vinen, Quantized Vortex Dynamics and Superfluid Turbulence. Berlin: Springer, 2001.

[4] G. K. Batchelor, The theory of homogeneous turbulence. Cambridge university press, 1953.

[5] T. Boder, M. H. Jensen, G. Paladin, and A. Vulpiani, Dynamical systems approach to turbulence. Cambridge University Press, 1998.

[6] R. J. Donnelly, Quantized vortices in helium II. Cambridge, UK: Cambridge University Press, 1991.

[7] W. Ebeling and I. L. Klimontovich, Selforganization and turbulence in liquids, vol. 2. BG Teubner Verlagsgesellschaft, 1984.

[8] U. Frisch, Turbulence: the legacy of AN Kolmogorov. Cambridge University press, 1995.

[9] K. Hutter and K. Jöhnk, Continuum methods of physical modeling: continuum mechanics, dimensional analysis, turbulence. Springer Science \& Business Media, 2013.

[10] J. Jovanovic, The statistical dynamics of turbulence. Springer Science \& Business Media, 2013.

[11] A. S. Monin and A. M. Yaglom, Statistical fluid mechanics, volume II: Mechanics of turbulence, vol. 2. Courier Corporation, 2013.

[12] S. K. Nemirovskii, "Quantum turbulence: Theoretical and numerical problems," Physics Reports, vol. 524, pp. 85-202, 2013. 
[13] S. Orszag, "Statistical theory of turbulence in fluid," $R$ Balin and JL Peube (London: Gordon and Breach), pp. 237-374, 1973.

[14] P. Asinari, M. Fasano, and E. Chiavazzo, "A kinetic perspective on $\mathrm{k}-\varepsilon$ turbulence model and corresponding entropy production," Entropy, vol. 18, no. 4, p. 121, 2016.

[15] L. Tisza, "Transport phenomena in helium II," Nature, vol. 141, p. 913, 1938.

[16] L. D. Landau, "The theory of superfluidity of helium ii," J. Phys., vol. 5, pp. 71-90, 1941.

[17] D. J. Melotte and C. F. Barenghi, "Transition to normal fluid turbulence in helium ii," Physical Review Letters, vol. 80, pp. 4181-4184, 1998.

[18] M. S. Mongiovì, D. Jou, and M. Sciacca, "Non-equilibrium thermodynamics, heat transport and thermal waves in laminar and turbulent superfluid helium," Physics Reports, vol. 726, pp. 1-71, 2018.

[19] C. F. Barenghi, S. Hulton, and D. C. Samuels, "Polarization of superfluid turbulence," Physical Review B, vol. 134, p. 275301 (4 pages), 2002.

[20] V. S. L'vov, S. Nazarenko, and O. Rudenko, "Bottleneck crossover between classical and quantum superfluid turbulence," Physical Review B, vol. 76, p. 024520, 2007.

[21] A. W. Baggaley, J. Laurie, and C. F. Barenghi, "Vortex-density fluctuations, energy spectra, and vortical regions in superfluid turbulence," Physical Review Letters, vol. 109, no. 20, p. 205304, 2012.

[22] A. W. Baggaley, L. Sherwin, C. F. Barenghi, and Y. Sergeev, "Thermally and mechanically driven quantum turbulence in helium ii," Physical Review B, vol. 86, no. 10, p. 104501, 2012.

[23] D. Jou, J. Casas-Vázquez, and G. Lebon, Extended Irreversible Thermodynamics. Berlin: Springer-Verlag, fourth ed., 2010.

[24] T. Ruggeri and M. Sugiyama, Rational extended thermodynamics beyond the monatomic gas. Springer, 2015.

[25] M. Sciacca, A. Sellitto, and D. Jou, "Transition to ballistic regime for heat transport in helium II," Physics Letters A, vol. 378, pp. 2471-2477, 2014.

[26] M. Mongiovì, "Superfluidity and entropy conservation in extended thermodynamics," Journal of Non-Equilibrium Thermodynamics, vol. 16, pp. 225-240, 1991.

[27] M. Mongiovì, "Extended irreversible thermodynamics of liquid helium II," Physical Review B, vol. 48, pp. 6276-6283, 1993.

[28] M. Tsubota, T. Araki, and W. Vinen, "Diffusion of an inhomogeneous vortex tangle," Physica B: Condensed Matter, vol. 329, pp. 224-225, 2003.

[29] S. K. Nemirovskii, "Diffusion of inhomogeneous vortex tangle and decay of superfluid turbulence," Physical Review B, vol. 81, p. 64512 (10 pages), 2010. 
[30] L. Saluto, D. Jou, and M. Mongiovì, "Vortex diffusion and vortex-line hysteresis in radial quantum turbulence," Physica B: Condensed Matter, vol. 440, pp. 99-103, 2014.

[31] L. Saluto, D. Jou, and M. Mongiovì, "Thermodynamic approach to vortex production and diffusion in inhomogeneous superfluid turbulence," Physica A: Statistical Mechanics and its Applications, vol. 406, pp. 272-280, 2014.

[32] M. Sciacca, M. Mongiovı, and D. Jou, "Alternative Vinen equation and its extension to rotating counterflow superfluid turbulence," Physica B: Condensed Matter, vol. 403, pp. 2215-2224, 2008.

[33] D. Jou, M. Mongiovì, and M. Sciacca, "Hydrodynamic equations of anisotropic, polarized and inhomogeneous superfluid vortex tangles," Physica D, vol. 240, pp. 249-258, 2011.

[34] S. K. Nemirovskii, "Nonuniform quantum turbulence in superfluids," Physical Review B, vol. 97, no. 13, p. 134511, 2018.

[35] D. Khomenko, L. Kondaurova, V. L'vov, P. Mishra, A. Pomyalov, and I. Procaccia, "Dynamics of the density of quantized vortex lines in superfluid turbulence," Physical Review B, vol. 91, p. 180504, 2015.

[36] A. W. Baggaley and J. Laurie, "Thermal counterflow in a periodic channel with solid boundaries," Journal of Low Temperature Physics, vol. 178, no. 1-2, pp. 35-52, 2015.

[37] S. Yui, K. Fujimoto, and M. Tsubota, "Logarithmic velocity profile of quantum turbulence of superfluid He 4," Physical Review B, vol. 92, no. 22, p. 224513, 2015.

[38] K. W. Schwarz, "Three-dimensional vortex dynamics in superfluid He 4: Homogeneous superfluid turbulence," Physical Review B, vol. 38, pp. 2398-2417, 1988.

[39] S. K. Nemirovskii, "Fluctuations of the vortex line density in turbulent flows of quantum fluids," Physical Review B, vol. 86, no. 22, p. 224505, 2012.

[40] S. B. Pope, "Turbulent flows," 2001.

[41] H. Tennekes and J. L. Lumley, A first course in turbulence. MIT press, 1972.

[42] J. Marshall and P. Naghdi, "Ii. determination of constitutive coefficients and illustrative examples," Phil. Trans. R. Soc. Lond. A, vol. 327, no. 1595, pp. 449-475, 1989.

[43] J. Marshall and P. M. Naghdi, "A thermodynamical theory of turbulence. i. basic developments," Phil. Trans. R. Soc. Lond. A, vol. 327, no. 1595, pp. 415-448, 1989.

[44] J. Marshall and P. Naghdi, "Consequences of the second law for a turbulent fluid flow," Continuum Mechanics and Thermodynamics, vol. 3, no. 1, pp. 65-77, 1991.

[45] P. Sancho, J. Llebot, and J. Fort, "Information-statistical extended thermodynamics and turbulent-induced heat flux inhibition," Open systems $\&$ information dynamics, vol. 6, no. 4, pp. 415-426, 1999.

[46] P. Sancho and J. Llebot, "Thermodynamic entropy and turbulence," Physica A: Statistical Mechanics and its Applications, vol. 205, no. 4, pp. 623-633, 1994. 
[47] H. Ozawa, S. Shimokawa, and H. Sakuma, "Thermodynamics of fluid turbulence: A unified approach to the maximum transport properties," Physical Review E, vol. 64, no. 2, p. 026303, 2001.

[48] H. Chen, S. A. Orszag, I. Staroselsky, and S. Succi, "Expanded analogy between Boltzmann kinetic theory of fluids and turbulence," Journal of Fluid Mechanics, vol. 519, pp. 301-314, 2004.

[49] H. Chen, S. Kandasamy, S. Orszag, R. Shock, S. Succi, and V. Yakhot, "Extended boltzmann kinetic equation for turbulent flows," Science, vol. 301, no. 5633, pp. 633-636, 2003.

[50] S. Ansumali, I. V. Karlin, and S. Succi, "Kinetic theory of turbulence modeling: smallness parameter, scaling and microscopic derivation of smagorinsky model," Physica A: Statistical Mechanics and its Applications, vol. 338, no. 3-4, pp. 379-394, 2004.

[51] C. M. Teixeira, "Incorporating turbulence models into the lattice-boltzmann method," International Journal of Modern Physics C, vol. 9, no. 08, pp. 1159-1175, 1998.

[52] R. Groll and H. Rath, "Thermodynamics/turbulence analogy modelling dissipating molecular gas flows for high knudsen numbers," PAMM, vol. 8, no. 1, pp. 10595-10596, 2008.

[53] L. Skrbek and K. Sreenivasan, "Developed quantum turbulence and its decay," Physics of Fluids (1994-present), vol. 24, p. 011301, 2012.

[54] M. Tsubota, K. Fujimoto, and S. Yui, "Numerical studies of quantum turbulence," Journal of Low Temperature Physics, vol. 188, pp. 119-189, 2017.

[55] L. Galantucci, M. Sciacca, and D. Jou, "The two-fluid extended model of superfluid helium," Accademia Peloritana dei Pericolanti Classe FF. MM. NN., vol. 97, no. S2, pp. A4-1-A4-15, 2019. 